\title{
Valores biométricos obtidos por ultra-sonografia dos tendões flexores e ligamentos acessório inferior e suspensório da região metacárpica palmar de cavalos Mangalarga Marchador
}

\author{
[Biometric values obtained by ultrasonography of flexor tendons and inferior accessory and suspensory \\ ligaments of the palmar metacarpal region in Mangalarga Marchador horses] \\ F.A. Aristizábal M. ${ }^{1}$, M.V. Souza ${ }^{2 *}$, J.R.M. Aranzales $^{3}$, J.I. Ribeiro Junior ${ }^{2}$ \\ ${ }^{1}$ Facultad de Medicina Veterinaria y Zootecnia - Universidad del Tolima - Ibagué - Tolima, Colombia \\ ${ }^{2}$ Universidade Federal de Viçosa \\ Av. P.H. Rolfs, s/n \\ 36570.000 - Viçosa, MG \\ ${ }^{3}$ Estudante de Pos-Graduação - Universidade Federal de Viçosa
}

\begin{abstract}
RESUMO
Determinou-se a área transversal (AT) dos tendões e ligamentos da região metacárpica palmar de 15 cavalos da raça Mangalarga Marchador. As áreas transversais do tendão do músculo flexor digital superficial (TFDS), do tendão do músculo flexor digital profundo (TFDP), do ligamento acessório inferior (LA) e do ligamento suspensório (LS) foram determinadas mediante ultra-sonografia, em sete zonas distintas (1A, 1B, 2A, 2B, 3A, 3B e 3C). Os valores médios da AT para TFDS foram: zona 1A, $1,07 \pm 0,13$; zona $1 \mathrm{~B}, 0,97 \pm 0,12$; zona $2 \mathrm{~A}, 0,77 \pm 0,08$; zona $2 \mathrm{~B}, 0,68 \pm 0,12$; zona $3 \mathrm{~A}, 0,74 \pm 0,10$; zona $3 \mathrm{~B}$, $0,86 \pm 0,10$; e zona $3 \mathrm{C}, 1,33 \pm 0,24$. Para TFDP foram: zona $1 \mathrm{~A}, 1,09 \pm 0,12$; zona $1 \mathrm{~B}, 0,99 \pm 0,14$; zona $2 \mathrm{~A}$, $0,72 \pm 0,11$; zona $2 \mathrm{~B}, 0,63 \pm 0,10$; zona $3 \mathrm{~A}, 0,71 \pm 0,14$; zona $3 \mathrm{~B}, 0,87 \pm 0,14$; e zona $3 \mathrm{C}, 1,39 \pm 0,20$. Para LA foram: zona $1 \mathrm{~A}, 1,17 \pm 0,14$; zona $1 \mathrm{~B}, 0,85 \pm 0,11$; zona $2 \mathrm{~A}, 0,73 \pm 0,12$; zona $2 \mathrm{~B}, 0,60 \pm 0,16$; e zona $3 \mathrm{~A}$, $0,42 \pm 0,15$. Para LS foram: zona $1 \mathrm{~A}, 0,82 \pm 0,14$; zona $1 \mathrm{~B}, 1,23 \pm 0,15$; zona $2 \mathrm{~A}, 1,21 \pm 0,15$; zona $2 \mathrm{~B}$, $1,18 \pm 0,14$; e zona $3 \mathrm{~A}, 1,20 \pm 0,31$. A AT de cada estrutura avaliada foi similar entre membros torácicos, considerando a mesma zona estudada. Não houve diferença significativa entre a AT do TFDS e a do TFDP.
\end{abstract}

Palavras-chave: eqüino, biometria, tendão, ligamento

\begin{abstract}
The transversal area (TA) of tendons and ligaments located in the palmar metacarpal region was studied in 15 Mangalarga Marchador horses without locomotor pathology. The superficial digital flexor tendon $(S D F T)$, deep digital flexor tendon (DDFP), accessory ligament of the deep digital flexor tendon (AL$D D F P)$, and suspensory ligament (TIOM) were examined by ultrasonography, at seven different zones $(1 A, 1 B, 2 A, 2 B, 3 A, 3 B$ e $3 C)$, to determine the TA. The mean TA values for SDFT were: zone $1 A$, $1.07 \pm 0.13$; zone $1 B, 0.97 \pm 0.12$; zone $2 A, 0.77 \pm 0.08$; zone $2 B, 0.68 \pm 0.12$; zone $3 A, 0.74 \pm 0.10$; zone $3 B$, $0.86 \pm 0.10$; and zone $3 C, 1.33 \pm 0.24$. For DDFT were: zone $1 A, 1.09 \pm 0.12$; zone $1 B, 0.99 \pm 0.14$; zone $2 A$, $0.72 \pm 0.11$; zone $2 B, 0.63 \pm 0.10$; zone $3 A, 0.71 \pm 0.14$; zone $3 B, 0.87 \pm 0.14$; and zone $3 C$, $1.39 \pm 0.20$. For $A L-D D F T$ were: zone $1 A, 1.17 \pm 0.14$; zone $1 B, 0.85 \pm 0.11$; zone $2 A, 0.73 \pm 0.12$; zone $2 B, 0.60 \pm 0.16$; and zone $3 A, 0.42 \pm 0.15$. For TIOM were: zone $1 A$, $0.82 \pm 0.14$; zone $1 B, 1.23 \pm 0.15$; zone $2 A$, 1.21 \pm 0.15 ; zone $2 B, 1.18 \pm 0.14$; and zone $3 A, 1.20 \pm 0.31$. The TA of each structure examined was similar between the
\end{abstract}

Recebido para publicação em 19 de setembro de 2003

Recebido para publicação, após modificações, em 10 de maio de 2004

*Autor para correspondência (corresponding author)

E-mail:msouza@ufv.br 
thoracic members in each of the studied zones. No significant difference was found among the TA of the SDFT and that of the DDFP.

Keywords: equine, biometry, tendon, ligament

\section{INTRODUÇ̃̃O}

A utilização intensiva de eqüinos em atividades esportivas tem aumentado a incidência de afeções do aparelho locomotor, em especial os membros torácicos, pois eles participam ativamente do amortecimento do peso durante a locomoção, o que possibilita o aparecimento de lesões nos tendões e ligamentos da região metacárpica palmar.

Na avaliação do aparelho locomotor é importante associar a inspeção e palpação às técnicas complementares de diagnóstico, como por exemplo, a ultra-sonografia. É uma técnica não invasiva que auxilia no diagnóstico e prognóstico de lesões em tendões e ligamentos. Henry et al. (1986) e McDiarmid (1995) mencionaram que para a devida interpretação dos achados ultrasonográficos é fundamental amplo conhecimento anatômico da região a ser avaliada.

Com a finalidade de padronizar o exame ultrasonográfico dos membros torácicos dos eqüinos, Hauser et al. (1985) dividiram a região metacárpica palmar em seis zonas, cada uma delas com aproximadamente $4,5 \mathrm{~cm}$ de comprimento. Esse sistema foi posteriormente modificado, sendo a região metacárpica dividida inicialmente em três zonas de igual comprimento (Genovese et al., 1986), a partir da base distal do osso acessório do carpo até a articulação metacarpofalangeana (região do ergot). Cada uma delas foi denominada de proximal à distal, como: zona 1, zona 2 e zona 3 , com aproximadamente $8 \mathrm{~cm}$ cada. As duas primeiras foram subdivididas em duas zonas (A e B) e a terceira incluiu a subdivisão $C$, perfazendo sete zonas, sendo a zona $1 \mathrm{~A} 0-4 \mathrm{~cm}$ distal ao osso acessório do carpo (DOAC); a zona $1 \mathrm{~B}, 4-8 \mathrm{~cm}$ DOAC; a $2 \mathrm{~A}, 8-12 \mathrm{~cm}$ DOAC; a $2 \mathrm{~B}, 12-16 \mathrm{~cm}$ DOAC; a $3 \mathrm{~A}, 16-20 \mathrm{~cm}$ DOAC; a $3 \mathrm{~B}, 20-24 \mathrm{~cm}$ DOAC e a $3 \mathrm{C}, 24-28 \mathrm{~cm}$ DOAC.

Para a determinação do tamanho das estruturas tendo-ligamentosas, as medidas podem ser tomadas no sentido dorsopalmar e lateromedial (Smith e Webbon, 1994). Porém como as lesões tendíneas geralmente se mostram de forma irregular, pode-se adotar critérios mais objetivos como a medição da área transversal (AT) (Marr et al., 1993; Smith e Webbon, 1994). A AT pode revelar aumento ou diminuição do tamanho que, segundo Hills (1996), depende do nível de treinamento e da raça do cavalo. Aumento de aproximadamente $20 \%$ sobre os valores de referência da raça são suficientes para suspeitar da presença de lesão (Smith e Webbon, 1994).

Poucos são os relatos realizados nessa área para eqüinos de raças nacionais. Assim, o objetivo deste estudo foi o de determinar, mediante ultrasonografia, o valor médio da área transversal (AT) dos tendões flexores e ligamentos acessório inferior e suspensório (músculo interósseo III), em sete distintas zonas da região metacárpica palmar de cavalos da raça Mangalarga Marchador, clinicamente sadios, e verificar a relação entre a AT dos tendões flexores.

\section{MATERIAL E MÉTODOS}

Foram utilizados 15 animais da raça Mangalarga Marchador, com idades entre quatro e 14 anos $(8,27 \pm 2,96), 13$ fêmeas e dois machos. $\mathrm{O}$ peso variou de 295 a $409 \mathrm{~kg}(353,66 \pm 33,14)$ e a altura de 141 a $152 \mathrm{~cm}(147,13 \pm 3,34)$. Os cavalos, utilizados para a reprodução, eram soltos em piquetes. Todos foram previamente submetidos a exame clínico (estático e dinâmico), com a finalidade de se descartar os que apresentassem alguma afecção clínica do aparelho locomotor. Em seguida determinou-se a área transversal $\left(\mathrm{cm}^{2}\right)$, mediante ultra-sonografia, dos tendões flexores e dos ligamentos da região metacárpica palmar dos membros torácicos.

O comprimento da região metacárpica palmar foi medido a partir da base distal do osso acessório do carpo até a articulação metacarpofalangeana, na região do ergot. $\mathrm{O}$ comprimento total variou de 24 a $25 \mathrm{~cm} \quad(24,6 \pm 0,43)$; a região foi posteriormente dividida em sete zonas $(1 \mathrm{~A}, 1 \mathrm{~B}$, $2 \mathrm{~A}, 2 \mathrm{~B}, 3 \mathrm{~A}, 3 \mathrm{~B}$ e $3 \mathrm{C}$ ), conforme descrito para os membros torácicos de cavalos Puro Sangue 
Inglês (Genovese et al., 1986). Entretanto, a dimensão de cada zona foi modificada tendo em vista o menor comprimento do metacarpo nos animais do estudo. Assim, foi considerado como zona $1 \mathrm{~A} \quad 0-3,5 \mathrm{~cm}$ distal ao osso acessório do carpo (DOAC); zona 1B, 3,5-7,0cm DOAC; zona $2 \mathrm{~A}, 7,0-10,5 \mathrm{~cm}$ DOAC; zona $2 \mathrm{~B}, 10,5-$ $14 \mathrm{~cm}$ DOAC; zona 3A, 14-17,5cm DOAC; zona $3 \mathrm{~B}, 17,5-21 \mathrm{~cm}$ DOAC e zona $3 \mathrm{C}, 21-24,5 \mathrm{~cm}$ DOAC.

Após tricotomia da região metacárpica palmar, foram obtidas imagens ultra-sonográficas, em tempo real, do tendão do músculo flexor digital superficial (TFDS), tendão do músculo flexor digital profundo (TFDP), ligamento acessório inferior (LA) e ligamento suspensório (LS). Para isso utilizou-se um aparelho de ultra-som, da marca Medison, modelo Sonovet $600^{\circledR}$, transdutor linear de 7,5 Mhz e um distanciador entre o transdutor e a pele, cujo objetivo foi reduzir o espaço entre eles e, conseqüentemente, a possibilidade de artefatos. As imagens foram capturadas pelo sistema magnético Magic Cap
(Sony). A área transversal de cada estrutura foi mensurada $\left(\mathrm{cm}^{2}\right)$ em cada uma das zonas definidas, utilizando-se o software QUANT v1.0.0.28, desenvolvido pelo Departamento de Solos da Universidade Federal de Viçosa.

Os dados foram tabulados e submetidos à análise estatística para a determinação da média e desvio-padrão de cada zona em ambos os membros torácicos. Para a comparação entre as médias dos membros torácicos dentro de cada estrutura e em cada zona, assim como entre os tendões flexores, utilizou-se o test $\mathrm{t}$ de Student para dados pareados, a $5 \%$ de probabilidade.

\section{RESULTADOS}

Os valores médios da $\mathrm{AT}$ do tendão do músculo flexor digital superficial, tendão do músculo flexor digital profundo, ligamento acessório inferior e ligamento suspensório são apresentados na Tab. 1.

Tabela 1. Valores $(\overline{\mathbf{X}} \pm \mathrm{DP})$ da área transversal $\left(\mathrm{cm}^{2}\right)$ do tendão do músculo flexor digital superficial (TFDS), tendão do músculo flexor digital profundo (TFDP), ligamento acessório inferior (LA) e ligamento suspensório (LS) segundo a zona da região metacárpica palmar

\begin{tabular}{|c|c|c|c|c|c|c|c|}
\hline & $\begin{array}{c}\text { Zona } \\
1 \mathrm{~A}\end{array}$ & $\begin{array}{c}\text { Zona } \\
1 \mathrm{~B}\end{array}$ & $\begin{array}{c}\text { Zona } \\
2 \mathrm{~A}\end{array}$ & $\begin{array}{c}\text { Zona } \\
2 \mathrm{~B}\end{array}$ & $\begin{array}{c}\text { Zona } \\
3 \mathrm{~A}\end{array}$ & $\begin{array}{c}\text { Zona } \\
\text { 3B }\end{array}$ & $\begin{array}{c}\text { Zona } \\
3 \mathrm{C}\end{array}$ \\
\hline TFDS & $\begin{array}{c}1,07 \mathrm{a} \\
0,13\end{array}$ & $\begin{array}{c}0,97 \mathrm{a} \\
0,12\end{array}$ & $\begin{array}{c}0,77 \mathrm{a} \\
0,08\end{array}$ & $\begin{array}{c}0,68 \mathrm{a} \\
0,12\end{array}$ & $\begin{array}{c}0,74 a \\
0,10\end{array}$ & $\begin{array}{c}0,86 \mathrm{a} \\
0,10\end{array}$ & $\begin{array}{c}1,33 a \\
0,24\end{array}$ \\
\hline TFDP & $\begin{array}{c}1,09 \mathrm{a} \\
0,12\end{array}$ & $\begin{array}{c}0,99 a \\
0,14\end{array}$ & $\begin{array}{c}0,72 \mathrm{a} \\
0,11\end{array}$ & $\begin{array}{c}0,63 \mathrm{a} \\
0,10\end{array}$ & $\begin{array}{c}0,71 \mathrm{a} \\
0,14\end{array}$ & $\begin{array}{c}0,87 \mathrm{a} \\
0,14\end{array}$ & $\begin{array}{c}1,39 a \\
0,20\end{array}$ \\
\hline LA & $\begin{array}{l}1,17 \\
0,14\end{array}$ & $\begin{array}{l}0,85 \\
0,11\end{array}$ & $\begin{array}{l}0,73 \\
0,12\end{array}$ & $\begin{array}{l}0,60 \\
0,16\end{array}$ & $\begin{array}{l}0,42 \\
0,15\end{array}$ & --- & --- \\
\hline LS & $\begin{array}{l}0,82 \\
0,14\end{array}$ & $\begin{array}{l}1,23 \\
0,15\end{array}$ & $\begin{array}{l}1,21 \\
0,15\end{array}$ & $\begin{array}{l}1,18 \\
0,14\end{array}$ & $\begin{array}{l}1,20 \\
0,31\end{array}$ & $\begin{array}{l}--- \\
---\end{array}$ & --- \\
\hline
\end{tabular}

Médias seguidas pela mesma letra na coluna não diferem entre si pelo teste t $(\mathrm{P}>0,05)$.

Valores médios de AT do TFDS e do TFDP foram menores nas zonas $2 \mathrm{~B}$ e $3 \mathrm{~A}$ e maiores nas extremidades, zonas $1 \mathrm{~A}$ e $3 \mathrm{C}$.

Houve redução gradual e contínua da área transversal do LA no sentido distal ao membro, diminuindo de forma marcada da zona $1 \mathrm{~A}$ até a $3 \mathrm{~A}$, e aumento da área de LS entre as zonas $1 \mathrm{~A}$ e $1 \mathrm{~B}$, com pouca variação nos valores de $\mathrm{AT}$ até a zona $3 \mathrm{~A}$, onde foi observado pela última vez, antes da sua divisão nos ramos lateral e medial.
Não foi observada diferença $(\mathrm{P}>0,05)$ nos valores médios de AT entre o tendão do músculo flexor digital superficial e profundo.

Também não foi observada diferença $(\mathrm{P}>0,05)$ nos valores médios de AT nos tendões (Tab. 2 e 3) e ligamentos (Tab. 4 e 5) entre os membros torácicos direito e esquerdo. A semelhança entre membros é melhor demonstrada na Fig. 1. 
Valores biométricos obtidos por ultra-sonografia...

Tabela 2. Valores $(\overline{\mathrm{X}} \pm \mathrm{DP})$ da área transversal $\left(\mathrm{cm}^{2}\right)$ do tendão do músculo flexor digital superficial segundo a zona e o membro avaliado

\begin{tabular}{lccccccc}
\hline \multirow{2}{*}{ Membro torácico } & Zona & Zona & Zona & Zona & Zona & Zona & Zona \\
& $1 \mathrm{~A}$ & $1 \mathrm{~B}$ & $2 \mathrm{~A}$ & $2 \mathrm{~B}$ & $3 \mathrm{~A}$ & $3 \mathrm{~B}$ & $3 \mathrm{C}$ \\
\hline \multirow{2}{*}{ Esquerdo } & $1,08 \mathrm{a}$ & $0,94 \mathrm{a}$ & $0,76 \mathrm{a}$ & $0,70 \mathrm{a}$ & $0,76 \mathrm{a}$ & $0,88 \mathrm{a}$ & $1,30 \mathrm{a}$ \\
& 0,15 & 0,12 & 0,07 & 0,12 & 0,12 & 0,12 & 0,23 \\
\multirow{2}{*}{ Direito } & $1,07 \mathrm{a}$ & $1,00 \mathrm{a}$ & $0,79 \mathrm{a}$ & $0,66 \mathrm{a}$ & $0,71 \mathrm{a}$ & $0,83 \mathrm{a}$ & $1,35 \mathrm{a}$ \\
& 0,11 & 0,12 & 0,09 & 0,11 & 0,08 & 0,08 & 0,26 \\
\hline
\end{tabular}

Médias seguidas pela mesma letra na coluna não diferem entre si pelo teste t $(\mathrm{P}>0,05)$.

Tabela 3. Valores $(\overline{\mathbf{X}} \pm \mathrm{DP})$ da área transversal $\left(\mathrm{cm}^{2}\right)$ do tendão do músculo flexor digital profundo segundo a zona e o membro avaliado

\begin{tabular}{lccccccc}
\hline \multirow{2}{*}{ Membro torácico } & Zona & Zona & Zona & Zona & Zona & Zona & Zona \\
& $1 \mathrm{~A}$ & $1 \mathrm{~B}$ & $2 \mathrm{~A}$ & $2 \mathrm{~B}$ & $3 \mathrm{~A}$ & $3 \mathrm{~B}$ & $3 \mathrm{C}$ \\
\hline \multirow{2}{*}{ Esquerdo } & $1,09 \mathrm{a}$ & $0,96 \mathrm{a}$ & $0,71 \mathrm{a}$ & $0,63 \mathrm{a}$ & $0,72 \mathrm{a}$ & $0,88 \mathrm{a}$ & $1,36 \mathrm{a}$ \\
& 0,15 & 0,12 & 0,07 & 0,09 & 0,15 & 0,12 & 0,17 \\
\multirow{2}{*}{ Direito } & $1,08 \mathrm{a}$ & $1,03 \mathrm{a}$ & $0,72 \mathrm{a}$ & $0,64 \mathrm{a}$ & $0,71 \mathrm{a}$ & $0,87 \mathrm{a}$ & $1,41 \mathrm{a}$ \\
& 0,07 & 0,16 & 0,15 & 0,11 & 0,14 & 0,16 & 0,23 \\
\hline
\end{tabular}

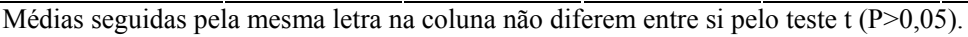

Tabela 4. Valores $(\overline{\mathbf{X}} \pm \mathrm{DP})$ da área transversal $\left(\mathrm{cm}^{2}\right)$ do ligamento acessório inferior segundo a zona e o membro avaliado

\begin{tabular}{lccccc}
\hline \multirow{2}{*}{ Membro torácico } & Zona & Zona & Zona & Zona & Zona \\
& $1 \mathrm{~A}$ & $1 \mathrm{~B}$ & $2 \mathrm{~A}$ & $2 \mathrm{~B}$ & $3 \mathrm{~A}$ \\
\hline \multirow{2}{*}{ Esquerdo } & $1,21 \mathrm{a}$ & $0,85 \mathrm{a}$ & $0,69 \mathrm{a}$ & $0,60 \mathrm{a}$ & $0,42 \mathrm{a}$ \\
& 0,14 & 0,10 & 0,11 & 0,13 & 0,11 \\
\multirow{2}{*}{ Directo } & $1,12 \mathrm{a}$ & $0,86 \mathrm{a}$ & $0,77 \mathrm{a}$ & $0,60 \mathrm{a}$ & $0,42 \mathrm{a}$ \\
& 0,14 & 0,13 & 0,11 & 0,19 & 0,19 \\
\hline
\end{tabular}

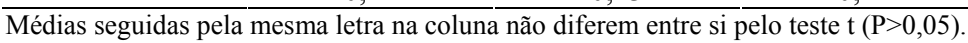

Tabela 5. Valores $(\overline{\mathrm{X}} \pm \mathrm{DP})$ da área transversal $\left(\mathrm{cm}^{2}\right)$ do ligamento suspensório segundo a zona e o membro avaliado

\begin{tabular}{lccccc}
\hline \multirow{2}{*}{ Membro torácico } & Zona & Zona & Zona & Zona & Zona \\
& $1 \mathrm{~A}$ & $1 \mathrm{~B}$ & $2 \mathrm{~A}$ & $2 \mathrm{~B}$ & $3 \mathrm{~A}$ \\
\hline \multirow{2}{*}{ Esquerdo } & $0,85 \mathrm{a}$ & $1,21 \mathrm{a}$ & $1,22 \mathrm{a}$ & $1,18 \mathrm{a}$ & $1,25 \mathrm{a}$ \\
& 0,16 & 0,17 & 0,15 & 0,14 & 0,19 \\
\multirow{2}{*}{ Directo } & $0,80 \mathrm{a}$ & $1,24 \mathrm{a}$ & $1,21 \mathrm{a}$ & $1,18 \mathrm{a}$ & $1,14 \mathrm{a}$ \\
& 0,12 & 0,14 & 0,15 & 0,15 & 0,40 \\
\hline
\end{tabular}

Médias seguidas pela mesma letra na coluna não diferem entre si pelo teste $\mathrm{t}(\mathrm{P}>0,05)$. 

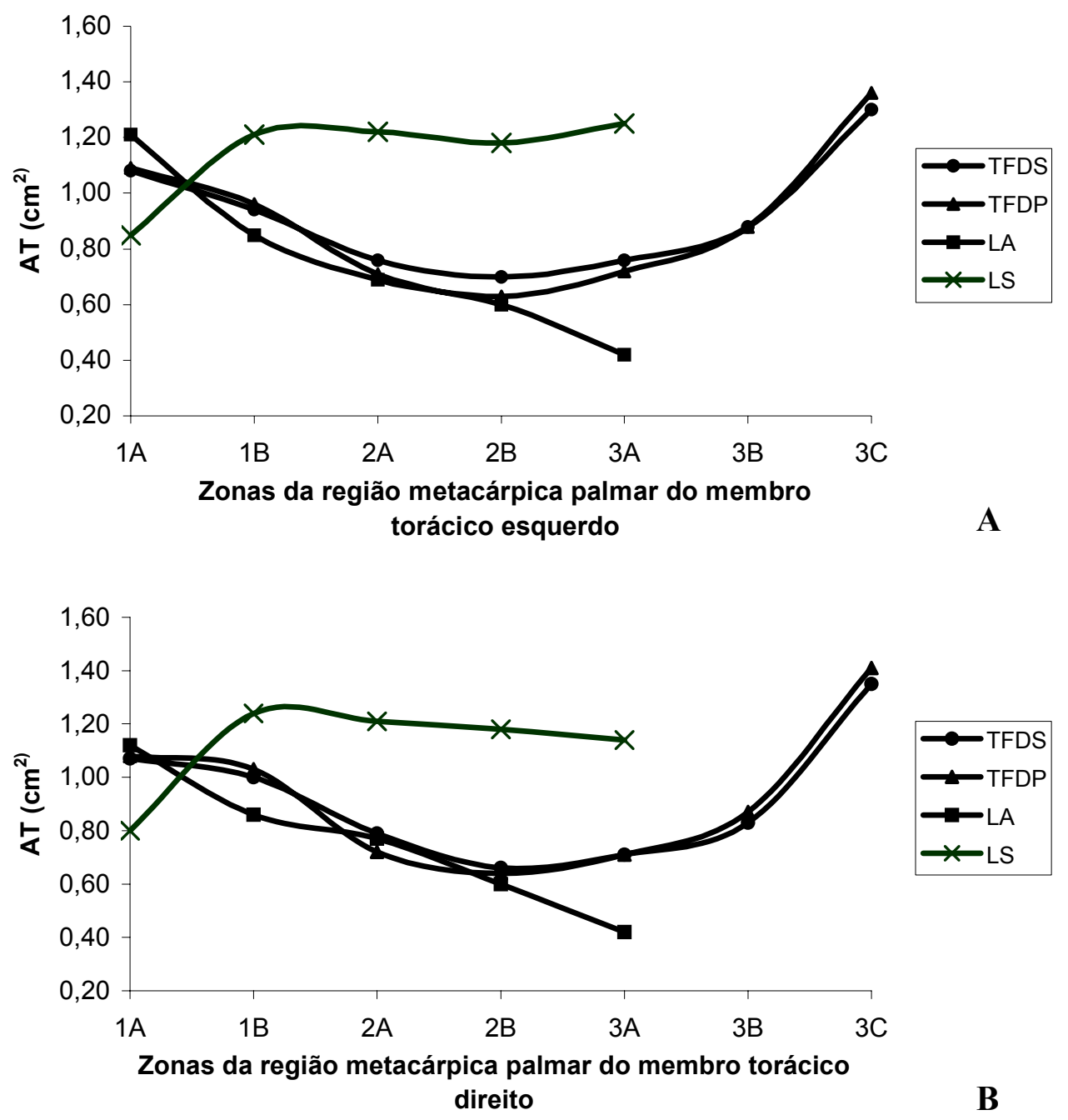

Figura 1. Variação das médias de área transversal (AT) do tendão do músculo flexor digital superficial (TFDS), tendão do músculo flexor digital profundo (TFDP), ligamento acessório inferior (LA) e ligamento suspensório (LS), segundo a zona estudada, nos membros torácico esquerdo (A) e torácico direito (B)

\section{DISCUSSÃO}

A utilização de um método digital para a obtenção da área transversal dos tendões flexores e ligamentos acessório inferior e suspensório proporcionou informação adequada e rápida sobre a espessura dessas estruturas. Os valores da AT obtidos indicaram apenas pequena variação entre os animais, semelhante ao descrito por Gillis et al. (1995), que mencionaram não existir associação entre AT das estruturas moles situadas na região metacárpica palmar e a idade ou sexo do animal. Hills (1996) constatou que as variáveis de maior influência na $\mathrm{AT}$ são a raça e o estágio do treinamento do cavalo. Para evitar esse problema foram incluídos neste estudo apenas animais submetidos a manejo e atividades semelhantes.

Pasin et al. (2001), ao trabalharem com 67 cavalos (21 de Hipismo, 23 Puro Sangue de Corrida e 23 Crioulos), observaram diferenças na AT entre os diversos tipos raciais. Os valores médios de AT dos tendões flexores obtidos neste estudo foram ligeiramente inferiores, com exceção da zona 3C do TFDS, aos encontrados 
por Pasin et al. (2001), em cavalos de hipismo e da raça Puro Sangue de Corrida. Com relação ao ligamento acessório inferior, os valores também foram inferiores, com exceção do encontrado na zona 1A. Estes resultados confirmam a importância em se obter parâmetros de referência para a raça.

A diminuição da AT nas zonas 2B e 3A dos tendões flexores (Tab. 1) também foi descrita por Gillis et al. (1995) para cavalos Puro Sangue Inglês. Segundo Williams et al. (1994), estas zonas, por apresentarem menor área do que a região mais central dos tendões flexores, são mais freqüentemente predispostas a lesões.

A semelhança entre os valores de AT obtidos nos membros torácicos direito e esquerdo também foi demonstrada por outros autores (Gillis et al., 1993; Hills, 1996). Segundo Reef (1998), em condições normais, não se espera diferença da AT entre membros contralaterais superior a $5 \%$. Essa semelhança entre membros permite a comparação de suas medidas, o que é particularmente importante diante de problemas que afetam unilateralmente os tendões e ligamentos, sendo possível detectar discretas variações de tamanho.

Segundo Spiers (1999), o ligamento suspensório pode ser observado mediante ultra-sonografia, até a zona $2 \mathrm{~B}$, antes da sua divisão nos ramos lateral e medial. Neste estudo, foi possível medir a AT desse ligamento inclusive na zona $3 \mathrm{~A}$, antes de sua bifurcação nos ramos mencionados. Tendo em vista que a distância entre as duas zonas é relativamente pequena, não se descarta a possibilidade de que tal achado seja decorrente do comprimento estabelecido para cada uma das sete zonas examinadas, que neste trabalho foi de $3,5 \mathrm{~cm}$ e não de $4,0 \mathrm{~cm}$, como considerado por Genovese et al. (1986) para cavalos Puro Sangue Inglês. Genovese e Rantanen (1998) relataram que a região mais distal do corpo do LS pode ainda ser detectada na zona $3 \mathrm{~A}$, aspecto verificado neste estudo.

Adaptações no tamanho de cada zona também foram realizadas por Pasin et al. (2001) para cavalos de Hipismo, Puro Sangue de Corrida e Crioulos. Segundo os autores, quando o comprimento do osso metacárpico III era de $30 \mathrm{~cm}$, cada zona correspondia a $4,5 \mathrm{~cm}$, e quando media $26 \mathrm{~cm}$ ou menos, correspondia a $3,7 \mathrm{~cm}$.
Gillis et al. (1995) mencionaram que o TFDS pode ser utilizado como referência na avaliação da forma e tamanho do TFDP e vice-versa. Essa afirmação pôde ser comprovada no presente estudo, já que os tendões flexores apresentaram valores de AT semelhantes.

\section{CONCLUSÕES}

O valor da área transversal das estruturas tendoligamentosas (TFDS, TFDP, LA e LS) situadas palmar à região metacárpica de animais da raça Mangalarga Marchador nos membros torácicos direito e esquerdo, por serem semelhantes, sugerem que o membro contralateral pode ser utilizado como referência. Os tendões flexores (TFDS e TFDP), por apresentarem valores médios de área transversal semelhantes, um deles pode servir de referência para o outro.

\section{REFERÊNCIAS BIBLIOGRÁFICAS}

GENOVESE, R.L.; RANTANEN, N.W. The superficial digital flexor tendon. In: RANTANEN, N.W., MCKINNON, A.O. (Eds.). Equine diagnostic ultrasonography. Baltimore: Williams \& Wilkins, 1998. Cap. 21, p.289-398.

GENOVESE, R.L.; RANTANEN, N.W.; HAUSER, M.L. et al. Diagnostic ultrasonography of equine limbs. Equine Vet. Educ., v.4, p.139-144, 1986.

GILLIS, C.; DENNIS, M.; MEAGHER, D.M. et al. Ultrasonographic cross sectional area and mean ecogenicity of the superficial and deep digital flexor tendons in 50 trained Thoroughbred racehorses. Am. J. Vet. Res., v.56, p.1265-1269, 1995.

GILLIS, C.L.; POOL, R.R.; CRAYCHEE, T.J. Ultrasonographically detected changes in equine superficial digital flexor tendons during the first months of race training. Am. J. Vet. Res., v.54, p.1797-1802, 1993.

HAUSER, M.L.; RANTANEN, N.W.; GENOVESE, R.L. Ultrasound anatomy and scanning technique of the distal extremities in the horse. In: ANNUAL CONVENTION OF THE AMERICAN ASSOCIATION OF EQUINE PRACTITIONERS, 31., 1985, Toronto, Canada. Proceedings... Toronto: 
AAEP, 1985. p.693-699 (Abstract).

HENRY, G.A.; PATTON, C.S.; GOBLE., D.O. Ultrasonographic evaluation of iatrogenic injuries of the equine accessory (carpal check) ligament and superficial digital flexor tendon. Vet. Radiol., v.27, p.132-140, 1986.

HILLS, A.C. Comparative ultrasonic study of normal tendinous and ligamentous structures of palmar metacarpus of Standardbred and Thoroughbred horses. In: ANNUAL CONVENTION OF THE AMERICAN ASSOCIATION OF EQUINE PRACTITIONERS, 42., 1996, Denver, Colorado. Proceedings... Colorado: AAEP, 1996. p.272-275 (Abstract).

MARR, C.M.; McMILLAN, I.; BOYD, J.S. et al. Ultrasonographic and histopathological findings in equine superficial digital flexor tendon injury. Equine Vet. J., v.25, p.23-29, 1993.
McDIARMID, A. Ultrasonography of the palmar metacarpus and pastern in the horse. Equine Pract., v.8, p.368-376, 1995.

PASIN, M.; BRASS, K.E.; ROSAURO, A.C. et al. Caracterização ultra-sonográfica dos tendões flexores em eqüinos: região metacarpiana. Arq. Fac. Vet., v.29, p.131-138, 2001.

REEF, V.B. Equine tendonitis discussion. $J$. Equine Med. Surg. Pract., v.20, p.17-21, 1998.

SMITH, R.K.W.; WEBBON, P.M. The crosssectional areas of normal equine digital flexor tendons determinated ultrasonographycally. Equine Vet. J., v.26, p.460-465, 1994.

SPIERS, V.C. Exame clínico de eqüinos. Porto Alegre: Artmed, 1999. 366p.

WILLIAMS, J.; MIYABAYASHI, T.; RUGGLES, A. Scintigraphic and ultrasonographic diagnosis of soft tissue in a Thoroughbred horse. J. Vet. Med. Sci., v.56, p.169-172, 1994. 